\title{
Flight dynamics simulation of formation flight for energy saving using LES-generated wake flow fields
}

\author{
Dennis Vechtel · Dietrich Fischenberg • \\ Jana Schwithal
}

\begin{abstract}
Wake vortices are an inevitable result of lift generation and can pose a threat to any aircraft, which accidentally encounters the wake of another aircraft. However, wake vortices can also be used in a beneficial way. Due to its rotational direction, the air flows upwards outside of the vortex pair, giving additional energy to any aircraft located in these regions. This method to save energy is used by migratory birds, resulting in these birds flying in the typical V-formations. This study deals with the question, whether it is possible with a standard autopilot (without a dedicated formation flight mode) to keep the aircraft's position accurately at a desired position in the wake flow field without accidentally encountering those areas of the wake where steady-state-flight is impossible, even in the presence of atmospheric disturbances (e.g. turbulence) and fluctuating vortex core positions. For this purpose, simulations were performed applying threedimensional flowfields generated with large eddy simulations (LES). Here, even with young vortices the target
\end{abstract}

This paper is based on a presentation at the German Aerospace Congress, September 13-15, 2016, Braunschweig, Germany.

D. Vechtel · D. Fischenberg · J. Schwithal

German Aerospace Center (DLR), Institute of Flight Systems, Lilienthalplatz 7, 38108 Braunschweig, Germany e-mail: dennis.vechtel@dlr.de

D. Fischenberg

e-mail: dietrich.fischenberg@dlr.de

J. Schwithal

e-mail: jana.schwithal@dlr.de sweet spot position varies in lateral and vertical direction with a magnitude of a few metres at a constant distance behind the generator aircraft. Hence, also the vortex induced forces and moments change continuously while flying at the same relative position to the leading aircraft. Preliminary simulations with an A320 flying in the wake of an A340, utilizing the regular autopilot of the comprehensive DLR A320 flight simulation model without a dedicated formation keeping mode, show that the autopilot does not accidentally encounter hazardous regions within the wake. This indicates that it could be sufficient for a formation keeping autopilot for civil transport aircraft to be designed as outer loop of the regular autopilot.

Keywords formation flight · large-eddy-simulation · fuel save $\cdot$ simulation study $\cdot$ flight control $\cdot$ autopilot
Abreviations
AIM Aerodynamic Interaction Model
ATRA Advanced Technologies Research Aircraft
AVES Air VEhicle Simulator
DLR German Aerospace Center
DME Distance Measuring Equipment
FL Flight Level
IAE International Aero Engines
ICAO International Civil Aviation Organisation
ILS Instrument Landing System
LES Large Eddy Simulation
NASA National Aeronautics and Space Administration
NDB Non-Directional Beacon
P2P Probabilistic two-phase model
RCR Roll Control Ratio 
VOR Very High Frequency Omnidirectional Radio Range

\section{List of symbols}

\begin{tabular}{|c|c|}
\hline$\Gamma$ & Circulation $\left(\mathrm{m}^{2} / \mathrm{s}\right)$ \\
\hline$\Gamma_{0}$ & Initial circulation $\left(\mathrm{m}^{2} / \mathrm{s}\right)$ \\
\hline$\Gamma_{5-15}$ & $\begin{array}{l}\text { Circulation averaged over radii between } 5 \mathrm{~m} \\
\text { and } 15 \mathrm{~m}\left(\mathrm{~m}^{2} / \mathrm{s}\right)\end{array}$ \\
\hline$b_{0}$ & Initial vortex spacing (m) \\
\hline$b$ & Wing span (m) \\
\hline$C_{l, \text { ind }}$ & Induced rolling moment (-) \\
\hline$C_{l, \xi_{\max }}$ & Maximum control rolling moment (-) \\
\hline$\varepsilon^{*}$ & Normalised eddy dissipation rate (-) \\
\hline$\Phi$ & Bank angle $\left({ }^{\circ}\right)$ \\
\hline$g$ & Earth’s gravitational constant $\left(\mathrm{m} / \mathrm{s}^{2}\right)$ \\
\hline$\rho$ & Air density $\left(\mathrm{kg} / \mathrm{m}^{3}\right)$ \\
\hline$r$ & Distance from vortex core (m) \\
\hline$r_{c}$ & Core radius (m) \\
\hline$m$ & Aircraft mass (kg) \\
\hline$N^{*}$ & Normalised Brunt-Väisalää frequency (-) \\
\hline$V_{T}$ & Tangential velocity (m/s) \\
\hline$V_{T A S}$ & True airspeed (m/s) \\
\hline$u, v, w$ & Velocity components $(\mathrm{m} / \mathrm{s})$ \\
\hline$\xi$ & Aileron deflection angle $\left(^{\circ}\right)$ \\
\hline$x, y, z$ & Cartesian co-ordinates (m) \\
\hline
\end{tabular}

\section{Introduction}

Whenever aircraft generate lift, they also produce wake vortices. This undesired but unavoidable result of the pressure differences between the upper and lower side of lift generating surfaces poses a potential threat to any aircraft encountering the wake of another aircraft unintentionally. The local flow velocities in the wake vortices can become very large and can lead to strong aircraft reactions of the encountering aircraft. This might involve injuries of passengers or crew as well as a temporary loss of control of the pilots. For these reasons minimum separation distances between different aircraft classes following or crossing each other were defined, by which hazardous wake encounters shall be prevented. The most prominent wake related separation scheme is that defined by ICAO [1].

However, wake vortices should not only be regarded as a threat, they can also be used in a beneficial way. Due to the rotational direction of the two vortices, the air flows downwards between them, while in the outer regions left and right of the vortex pair the air flows upwards (Fig. 1). This upwind gives additional energy to any aircraft located in these regions. The energy spent by a flying aircraft for the generation of its own wake vortices, the induced drag, can be partly harvested by another aircraft flying in the vortices' upwind regions. This method to save energy is also performed by migratory birds, resulting in the typical $\mathrm{V}$-formations of many birds, such as e.g. geese [2,3].

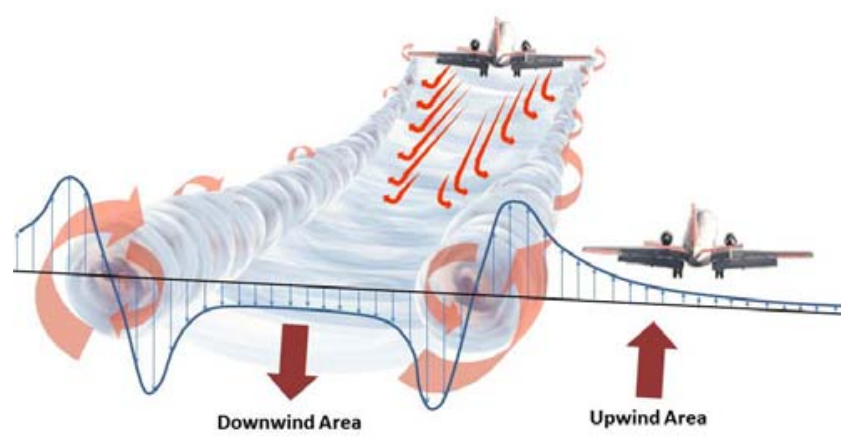

Fig. 1 Vortex flow field and beneficial regions for formation flight

One can understand the mechanism of energy saving by flying in the vortices' upwind by comparing it with flying a glider aircraft in a thermal or a hang-glider in an upwind in front of a mountain. In these cases the aircraft flies in an upwards moving air, such that for maintaining the same altitude the aircraft needs to "sink" in the rising air. For a propelled aircraft this reduces the necessary propulsion effort, hence the fuel burn. From a flight mechanical point of view the flight in an upwind field results in a forward inclination of the aerodynamic force vector.

After the phenomenon was thoroughly investigated regarding birds in the 1970s [4,5], theoretical analyses were performed in the 1980s and 1990s for the application of formation flight for the purpose of fuel saving of fixedwing aircraft [6,7]. In the early 2000s the US Air Force and NASA conducted flight tests with F/A-18 aircraft in close formation and confirmed a reduction in fuel burn of $5 \%$ to $10 \%$ for narrow lateral separations between leader and follower aircraft $[8,9]$. Quite recently, the US Air Force and Boeing conducted a comprehensive flight test campaign together with numerical aerodynamic analyses for C17 aircraft, which showed fuel savings in the same magnitude $[10,11]$.

Theoretical studies for analysis of the saving potential of formation flight are typically performed for a specific longitudinal position of the follower aircraft behind the generator. At different lateral and vertical positions of the follower (relative to the generator) the fuel saving varies due to variable intensity of the wake's impact. The location with maximum fuel savings is usually called sweet-spot. 
An example of such theoretical studies is given in Fig. 2 [12].
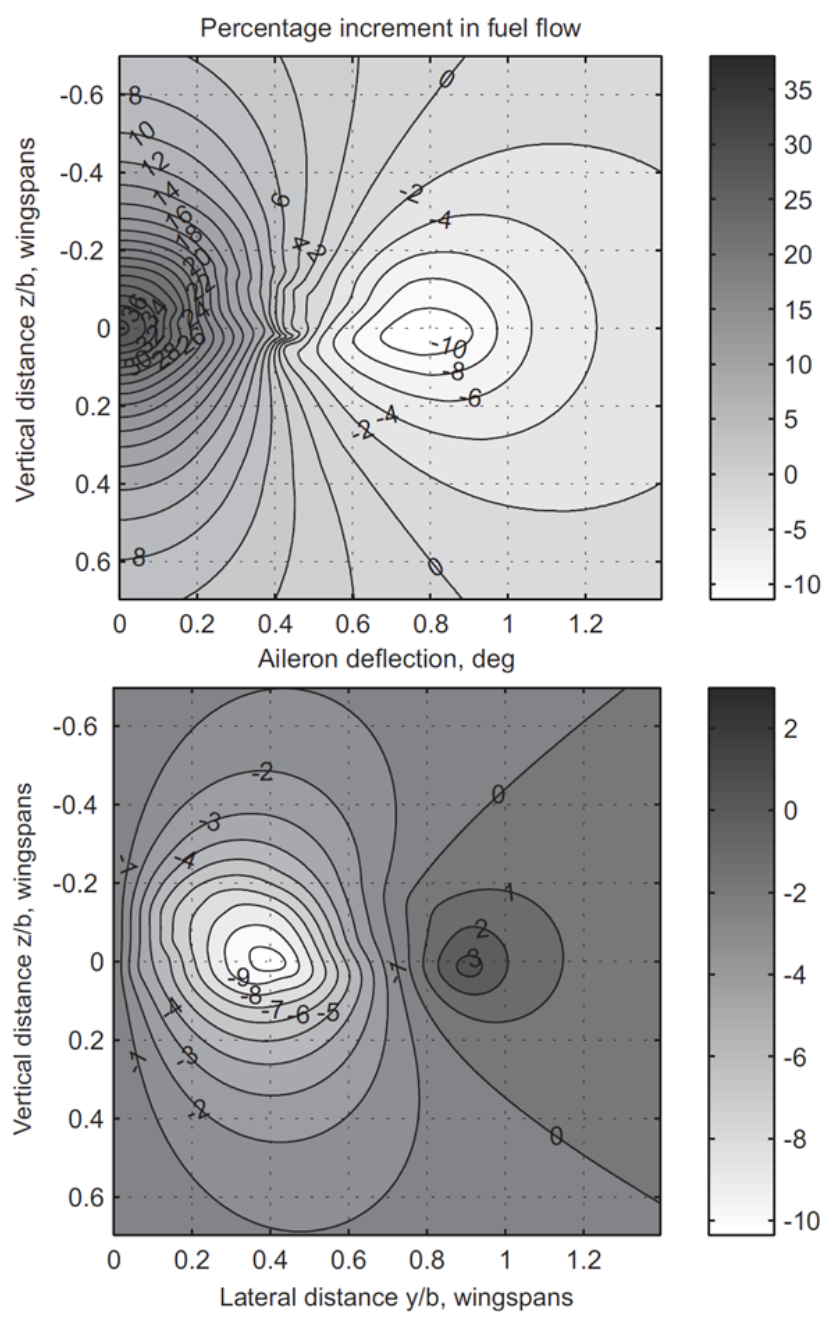

Fig. 2 Results from formation flight simulations from Kaden [12]

Fig. 2 shows trim results from formation flight simulations with two VFW 614 aircraft, where the follower aircraft is located five wingspans $\left(b_{V F W 614}=21.5 \mathrm{~m}\right)$ behind the generator. The percental increment in fuel flow and the aileron deflection were evaluated for different lateral and vertical positions of the following aircraft's center of gravity in the plane behind the leading aircraft placed at the origin of ordinates (positions normalised with the aforementioned wingspan of the VFW 614).

For steady flight in an inhomogeneous flow field, deflections of the control surfaces are necessary in order to keep the aircraft at a constant position in the flow field. As can be seen in Fig. 2, the area with maximum fuel saving is located in that region where the aileron deflection, hence the impact on rolling moment, is relatively low. The location of this region in relation to the generator aircraft depends on the geometries of leader and follower aircraft. However, regardless of the sweet spot's exact location (relative to the leader), it is typically close to the region with large gradients in the induced rolling moment as can be seen in previous studies such as that of Kaden [12] shown in Fig. 2 or the simulations shown in the following sctions in this paper. A flight in this region would indeed maximise fuel savings, but as the distribution of induced rolling moment leads to an unstable flight condition, flying in this region might be like balancing on a knife's edge. Any disturbance, which leads the follower aircraft closer to the vortices, would result in an induced rolling moment leading the aircraft even nearer towards the vortices and the downwind area. On the other side any disturbance, which leads the follower further away from the vortices, would result in an induced rolling moment leading the aircraft even further away from the vortices. This unstable situation may result in harsh requirements to the autopilot for position keeping. The required accuracy of positioning the aircraft relatively to the vortices is in the magnitude of a few metres.

Many formation flight studies in the past used idealised models of vortices. This means the vortex lines are ideally straight with no changes in flight direction. Hence, the optimisation task for formation flight is reduced to a quasi2D scenario (2.5D). With such ideal vortex models it might indeed be possible to fly exactly at the optimal position. However, in reality atmospheric disturbances, such as light wind shear or turbulence, can result in perturbations of the vortex lines even at cruise flight levels. This might even occur right after the vortices have rolled up and no instability effects, which occur during the decay, have evolved yet (Fig. 3 shows a real flight example for this).

During the flight test studies with the C-17 aircraft, tests were conducted by flying closely to the vortex cores and even crossing the vortices. Different flight patterns in the vicinity of the vortices and through the vortex cores were performed in order to validate the prediction of the vortex position and to evaluate the fuel saving potential at different relative lateral positions. Most of the time the follower aircraft flew at lateral positions outside the sweet spot in order to lower structural stresses on the aircraft and improve the ride quality [10].

The C-17 possesses a dedicated autopilot mode for formation flight, which was adapted for the flight tests in order to maintain a specific relative position to the vortices. As present civil aircraft usually do not have such a formation flight autopilot mode, the question raised here is, whether it is also possible with a standard autopilot, under the presence of atmospheric disturbances and fluctuating 
vortex core positions, to keep the aircraft's position accurately in the optimal region of the fuel saving sweet spot or whether the aircraft needs to position further away from the sweet spot, giving up some percentage points of fuel saving, for reasons of flight safety.

For this purpose, the simulation study presented here applies non-uniform, three-dimensional flow fields generated with large eddy simulations (LES), which represent perturbed vortices like they can occur under real flight conditions. It should be noted that for the time being passenger comfort is not in the scope of investigation as the used aircraft simulation model only accounts for the rigidbody motion. However, for a meaningful investigation of passenger comfort, flexibility of the aircraft needs to be considered. Also, the reader should keep in mind that the work presented here is not aiming for the evaluation of boundaries for any metric. Neither the optimal region for formation flight within the flow field, nor the thresholds for the minimal safe distance to the vortex cores should be given here. The study investigates the influence of nonuniform flow fields on the existing autopilot's ability to maintain the flight state at or in vicinity to the sweet spot without unintentionally encountering those areas of the flow field where the induced forces and moments cannot be counteracted.

\section{Simulation}

For evaluation of fuel saving and induced rolling moment at a specific position in the wake's flow field no dynamic simulation is required. Trim calculations were performed at different positions within the wake's flow field. Dynamic simulations were performed for the evaluation of the aircraft's behaviour during flight in the perturbed flow field.

The simulation models used in this study have been developed at the DLR Institute of Flight Systems and comprise the flight dynamics model of the DLR research aircraft A320 ATRA (Advanced Technologies Research Aircraft) as the follower aircraft in the formation. The components of the simulation framework are briefly described in the following sections, namely the dynamic model of the aircraft simulation and the flight control system, the vortex flow fields and the aerodynamic interaction model, which is required for the calculation of the vortex induced forces and moments acting on the trailing aircraft.

\subsection{Aircraft and flight control system model}

The simulation model was developed and upgraded continuously since the A320 ATRA came into service at
DLR in 2006. As the model is validated using flight test data from the real aircraft, the model accuracy is considered acceptable for scientific simulation purposes (without the intention to use it for pilot training) [13]. However, most requirements for pilot training simulations are met by the model within the tolerance range $[13,14]$.

The aircraft model comprises an aerodynamic model based on stability and control derivatives identified from flight test data, a stall and ground effect model, a simplified propulsion model of the IAE V2500 engines based on look-up tables, a landing gear model, sensor models of the radio altimeters, air data system (pressure sensors, wind vanes, etc.), inertial reference system and navigation sensors (DME, NDB, VOR, ILS, etc.), as well as simplified actuator models for all control surfaces (second order actuator with time delay).

The ATRA simulation includes a flight control system, which comprises direct and normal control laws for manual flight as well as a standard autopilot (understood here as an autopilot without a dedicated formation flight mode). The design of the controllers was based on the Airbus flight control system philosophy. All flight modes of the A320 were implemented. The autopilot comprises all modes for automatic flight of the A320 using selected command values (such as altitude acquire and hold, heading / track acquire and hold, open descent and climb or descent / climb with selected vertical speed etc.) but also enables managed flight based on flight-plan-related command values. In this study the autopilot was used for the dynamic simulations in altitude and heading hold mode together with the autothrust system in speed mode. Also, the normal law, which compensates roll disturbances, was used without a pilot model.

The reader should be aware that the autopilot behaviour on an external disturbance, such as a wake vortex, could not directly be validated by means of flight test data as for such a validation the external disturbance has to be known. The development of the controllers for the flight control system and the autopilot simulation was mainly based on manuals available to aircraft operators $[15,16]$. The autopilot behaviour on control inputs was validated by means of flight data from the real aircraft. However, the aircraft response in case of an external disturbance is not necessarily fully representative of the original A320.

\subsection{Vortex flow field models}

For reasons of comparison different wake vortex models were used in this study. In order to compare the simulation results with results from other studies straight vortices were used for the quasi-2D case. For investigation on the 
influence of vortex perturbations, like they occur under real flight conditions, a pre-computed three-dimensional flow field was used. Both models are described in the following.

The three-dimensional flow field was generated with large eddy simulations (LES). The LES were conducted by the DLR Institute of Atmospheric Physics within the frame of a DLR-internal project called "Weather and Flying" between 2008 and 2011 [17].

The wake generating aircraft is an A340 regarding mass and wing span. This aircraft pairing with A340 as leader and A320 as follower is unusual for formation flight, as most previous studies assumed the same aircraft type for both, leader and follower. However, when comparing the simulation results with different aircraft types (s. Figs 5 and 6) with those with the same aircraft type (s. Fig. 2) one can observe that the general effects are the same. With a much heavier generator aircraft than the follower, the maximum values of saved fuel and rolling moment impact are just larger than compared with other studies. This is due to the fact that here the generator aircraft is vastly heavier than the follower, whereas typically both aircraft have the same or a comparable mass.

The LES dataset with the youngest vortex available from the "Weather and Flying" project is a flow field of a 16 seconds old wake. A usual way to express the vortex age is the normalised vortex age $t^{*}$

$$
t^{*}=\frac{t}{2 \cdot \pi \cdot \frac{b_{0}^{2}}{\Gamma_{0}}}
$$

The normalised vortex age of the flow field used here is $t^{*}=0.53$. The atmospheric conditions for the LES were moderate turbulence (normalised eddy dissipation rate $\varepsilon^{*}=0.23$ ) and neutral thermal stratification (normalised Brunt-Väisalää frequency $N^{*}=0$ ). These conditions play a bigger role only during the later vortex decay (for the circulation decrease and vortex deformation). For such early vortex stages as considered for formation flight the influence of turbulence and stratification is less relevant. Therefore, the exact values are just mentioned for reasons of completeness. With a cruise Mach number of 0.78 at a cruise altitude of 37,000 ft (FL370) this vortex age represents a separation distance between leader and follower aircraft of $2 \mathrm{~nm}$. In reality the separation distance during formation flight would probably be smaller but as the circulation decreases only slowly after the vortex generation the vortex strength is comparable, regardless if the separation is e.g. $0.5 \mathrm{~nm}$ or $2 \mathrm{~nm}$. The most important issue is that at this vortex age no decay-related disturbance of the vortices, such as the Crow-instability, has evolved yet.
Therefore, the vortices can be regarded as straight, even though the vortex lines are not perfectly straight. The perturbations of the vortex line only result from the underlying atmospheric turbulence. However, it can be expected that the perturbations at this relatively large vortex age (for the pupose of formation flight) are larger than at younger vortex ages, so that the simulations presented here can be considered especially conservative. The atmospheric conditions are considered to enhance the representativeness of this simulation study.

The mean circulation, averaged over radii between $5 \mathrm{~m}$ and $15 \mathrm{~m}$ from the vortex line, is $\widetilde{\Gamma}_{5-15}=451 \mathrm{~m}^{2} / \mathrm{s}$, the maximum is $\Gamma_{5-15, \max }=462 \mathrm{~m}^{2} / \mathrm{s}$. It must be mentioned here, that originally the LES were conducted for the approach flight phase. This means that the generator's airspeed was $75 \mathrm{~m} / \mathrm{s}$ with an air density of $\rho=1.225 \mathrm{~kg} / \mathrm{m}^{3}$. Nevertheless, the flow fields can be applied to the cruise flight phase as the much lower air density at cruise flight levels, the much greater airspeed and usually greater mass of the generator compensate each other quite well, and therefore result in an initial circulation of comparable magnitude. The initial circulation of a wake vortex is

$$
\Gamma_{0}=\frac{m \cdot g}{\rho \cdot b_{0} \cdot V_{T A S}}
$$

With a cruise Mach number of 0.78 at FL370, an aircraft mass of $180 \mathrm{t}$ and an initial vortex spacing of $47.36 \mathrm{~m}$ (given the wing span of the A340 and $b_{0}=\frac{\pi}{4} \cdot b$ for an elliptic lift distribution) the initial circulation is $\Gamma_{0}=465 \mathrm{~m}^{2} / \mathrm{s}$. Another parameter that influences the vortex flow field is the core radius. This can also be considered as independent from the flight phase as it was identified to only depend on the vortex generator's wing span [18]. This shows that the LES used here represent cruise flight conditions well.

Fig. 3 shows the LES-generated flow field at a vortex age of $16 \mathrm{~s}$. The left side of the figure depicts the isosurface of a flow velocity of $7 \mathrm{~m} / \mathrm{s}$ of the numerical flow field. At the right side of Fig. 3 a photo taken from DLR flight tests exemplarily shows the roll-up of a B747's wake. The photo clearly outlines that even right after the vortices have rolled-up perturbations of the vortex lines may occur, possibly from turbulence or light wind shear. Fig. 3 shows, that the shape of the used LES vortex flow fields is representative for a realistic vortex shape for the envisaged purpose of formation flight. 


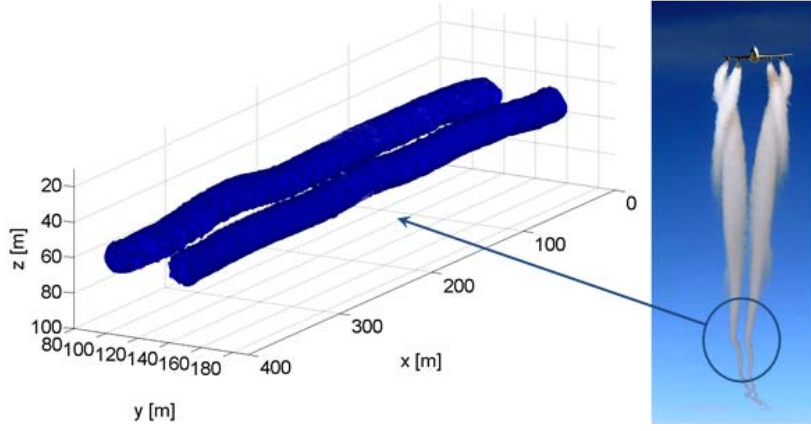

Fig 3. Shape of LES-generated vortex flow field (left) and photo of real vortex shape [photo: DLR]

The vortex dataset was implemented in the simulation as a 3D flow field using look-up tables with a linear interpolation method for the three velocity components $u, v$ and $w$ as a function of the spatial positions $x, y$ and $z$. The spatial resolution of the look-up table data is $1 \mathrm{x} 1 \mathrm{x} 1$ metre, whereupon the core radius $r_{c}$ is about $3.5 \mathrm{~m}$ at this vortex age. Hence, the vortex core always includes a sufficient number of data points to be properly represented. As can be seen in Fig. 3 the length of the LES flow field is $400 \mathrm{~m}$. One basic property of LES is its periodic boundaries, which means that the output of one boundary acts as input to the opposite one. For this reason the LES flow field can be connected in series resulting in an infinitely long flow field with the repeated shape of the vortices at one age. Flying through this windfield in vortex direction means that the vortex age remains unchanged, which is indeed the case if generator and follower aircraft fly with the same airspeed.

For reasons of comparison with other studies, the same results were generated using an analytical model of straight vortices. These vortices were modelled by using the radial distribution model of the tangential velocity by Burnham and Hallock [19], or Rosenhead [20] respectively. The flow velocities were calculated by superimposing two single, counter-rotating, straight and infinitely long vortices. The tangential velocity of a vortex of infinite length to both sides is calculated by

$$
V_{T}=\frac{\Gamma}{2 \pi} \frac{r}{r^{2}+r_{c}{ }^{2}}
$$

with the vortex circulation $\Gamma$, the core radius $r_{c}$ (at which the tangential velocity has its maximum) and the radial distance from the vortex centre $r$. Fischenberg identified the core radius by means of flight test data to be $3.5 \%$ of the wing span $b$ of the generator aircraft [18] using this model for the tangential velocity. This leads to a slightly smaller core radius $\left(r_{c}=2.1 \mathrm{~m}\right)$ than that from the LES $\left(r_{c}\right.$ ranging between $2,9 \mathrm{~m}$ and $4.1 \mathrm{~m}$ ). Assuming an elliptical spanwise lift distribution the initial vortex spacing $b_{0}$ is $\pi / 4$ of the wing span $b$ (hence, $b_{0}=47.4 \mathrm{~m}$ ), which is assumed to be constant for the considered vortex ages for formation flight.

As mentioned above the circulation of the LES flow fields is not constant. Therefore, the circulation of the analytical vortices is calculated with the deterministic version of the P2P two-phase decay model [21]. With the geometry and a typical cruise mass of the A340 the circulation used for the straight vortices at a vortex age of $16 \mathrm{~s}$ is $\Gamma=468 \mathrm{~m}^{2} / \mathrm{s}$. This value is comparable to the range of circulation of the LES flow fields. This way the energy of the simple analytical vortices is comparable to that of the LES vortices.

\subsection{Aerodynamic interaction model}

Besides the vortex flow field models an aerodynamic interaction model (AIM) is required for the calculation of vortex-induced forces and moments acting on the following aircraft. As the aircraft model of the A320 ATRA already incorporates an accurate aerodynamic model, the AIM used for the formation flight simulations is a deltamodel calculating only the forces and moments resulting from the additional angle of attack and sideslip angle due to the vortex flow.

The AIM uses the so-called strip method, where lift and side force generating surfaces (wings, stabilisers and fuselage) of the aircraft are divided into sections for which the vortex influence is determined (Fig. 4). For strips of horizontal planes an additional lift force and for strips of vertical planes an additional side force is determined. The vortex-induced forces and moments are fed into the equations of motion of the aircraft model.

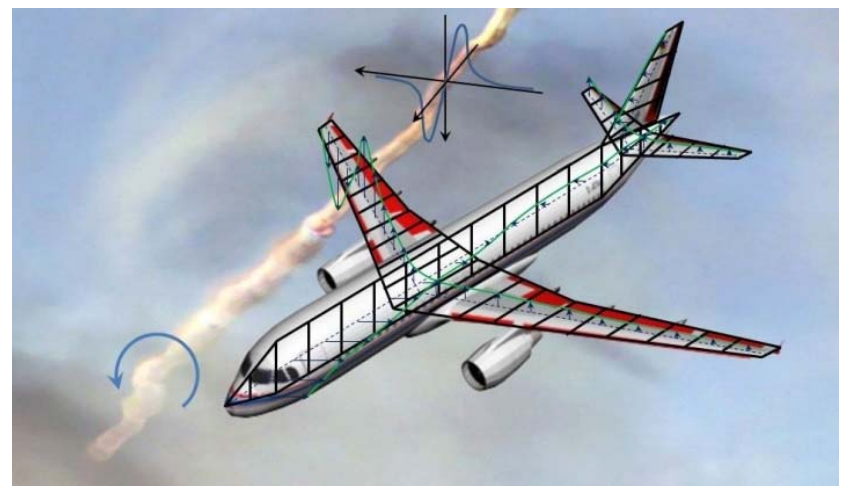

Fig. 4 The strip method [22] 
The method was deemed feasible by Barrows [23], verified against wind tunnel tests by de Bruin [24] and further validated using real flight test data by Fischenberg [18] and Jategaonkar [25]. Furthermore, this method was applied in various simulation studies in the past [26-30] and is accepted to provide vortex-induced forces and moments of acceptable accuracy.

Originally, the AIM was developed as a 5-DoF model, neglecting any drag effects. This might be sufficient for simulation of wake encounters, which usually last for only a few seconds, mainly resulting in rolling motion of the encountering aircraft. However, for evaluation of fuel saving from long lasting formation flight the accounting for drag is mandatory. So, this degree of freedom was modelled for each strip in a simplified manner as a linear approximation of the drag polar of the aircraft (no local polar for each strip) at the considered operating point, whose coefficients were defined based on the same wake encounter flight test measurements as already used in [28]. Also, drag coefficients of the control surfaces were identified by those flight test results.

\section{Results}

The most important measure for analysis of formation flight is the fuel flow or the saved fuel in comparison to the nominal fuel burn without formation flight. Another important issue to be considered is the impact of the vortices on the motion of the following aircraft. In case of formation flight, when both aircraft fly with the same azimuth, the rolling moment induced by the vortex flow field is the most relevant impact besides the induced lift. Coming too close to the vortices the induced rolling moment can exceed the controllability of the aircraft. Therefore, a too large rolling moment impact has to be avoided for safe formation flight. In order to quantify the rolling moment impact, the so-called Roll Control Ratio $R C R$ is used here [31]. The $R C R$ is the ratio between the vortex-induced rolling moment and the maximum rolling moment which can be applied by a full deflection of all roll motivators (such as ailerons and roll spoilers)

$$
R C R=\frac{C_{l, i n d}}{C_{l, \xi_{\max }}}
$$

$R C R$ is a good indication for the necessary control effort in the roll axis, which is a relevant issue when flying in the wake's flow field. $R C R$ was first introduced by Crow in 1970 [32] and is since then often used as hazard metric for the severity assessment of wake encounters and good expe- riences were made in this area with $R C R$ in the past [26,27,29-31].

The two metrics used here, the saved fuel flow and $R C R$, represent the two sides of the medal for formation flight. Whilst the saved fuel flow is the parameter to be maximised, the $R C R$ represents the potential threat which is posed by the wake and should be avoided. For safe formation flight, these two factors must be balanced.

One thing the reader should be aware of is that all simulations described here were conducted without wind. This might indeed sound like an operationally irrelevant case as in cruise flight wind is almost always present. However, the presence of wind does not change the overall geometry of the formation flight especially for keeping a relative position besides the vortices. This can be seen in Fig. 5 . no wind

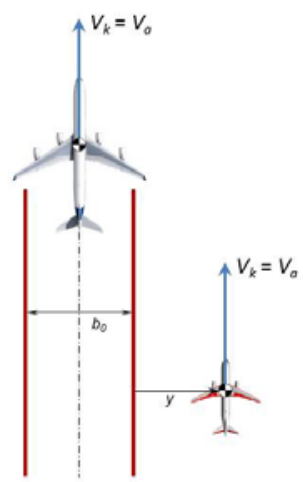

wind

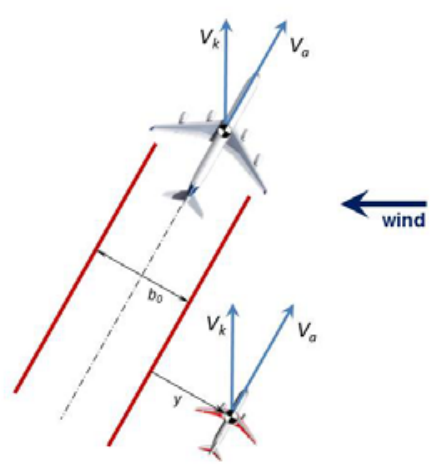

Fig 5. Geometry of the formation flight situation with and without wind

Under the presence of wind both aircrafts' heading point into the wind. As the wake vortices drift in leeward direction due to the wind they point into the direction of the generator's heading as well. The only difference is that vectors of ground speed and airspeed do not point into the same direction anymore. In order to control the desired position relative to the vortex $y$ the autopilot of the follower aircraft could use either heading or track command. Practically, one would probably choose a track command for this purpose, but generally it would also work with a heading command to control the follower's position in the wake's flow field. For this reason and for reasons of simplicity the following simulations were conducted without wind, keeping in mind that this does not generally change the situation under real flight conditions with wind prevailing. 


\subsection{Perfectly straight vortices}

For a general understanding of the major effects of formation flight straight vortices were applied in the simulation. Fig. 6 shows the spatial distribution of the fuel flow reduction in lateral and vertical direction in relation to the generator aircraft and the vortices. The aircraft depicted here are drawn to scale in order to give an impression of the relative scale between both aircraft and the vortex flow field. The white area in Fig. 6 is that area behind the generator aircraft where no steady-state flight is possible, either due to the downwash (leading to reach the thrust limitation) or the rolling moment (exceeding the roll control authority) of the vortices or a combination of both. In this area the trim routine of the A320 simulation could not find a solution as no steady-state flight is possible. Therefore, no data exist within the white area. The reader should be aware that in the following figures no sinking of the vortices is considered. Under real flight conditions the vortices would have indeed descended a considerable distance $2 \mathrm{~nm}$ or $16 \mathrm{~s}$ behind the leader aircraft. As for the aircraft simulation this effect is not relevant vortex sinking is neglected here.

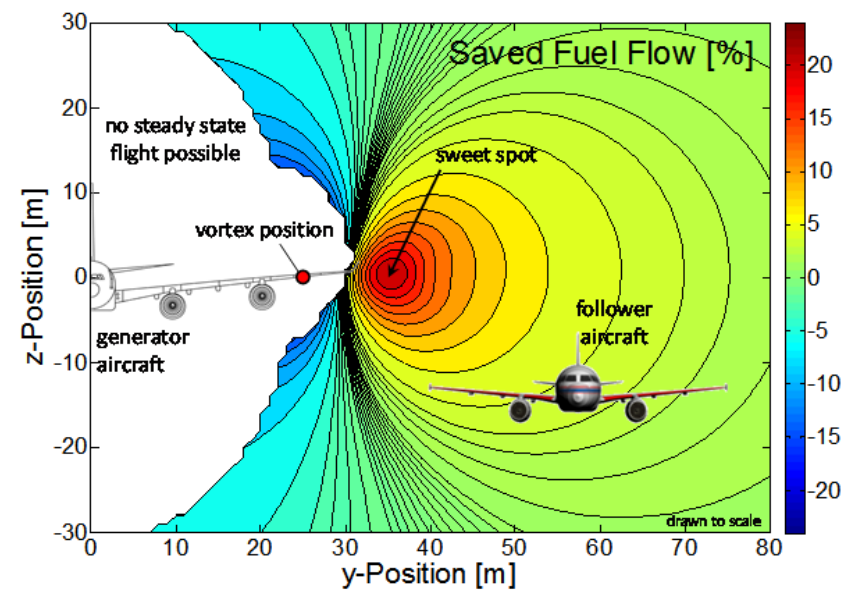

Fig. 6 Spatial distribution of fuel flow reduction with straight analytical vortices (A320-type $2 \mathrm{~nm}$ behind A340type at FL370, Ma 0.78)

One can observe in Fig. 6 that in this case the sweet spot (i.e. the position with maximum fuel saving) is located at a lateral position of about $35 \mathrm{~m}$ from the symmetry plane of the generator aircraft. This position is only about $10 \mathrm{~m}$ next to the vortex core, which is located at a lateral position of about $25 \mathrm{~m}$. The sweet spot is only approximately $5 \mathrm{~m}$ next to the area where no steady state flight is possible. The maximum reduction of the fuel flow of about $20 \%$ is indeed enormous, but it is caused by the large differences in aircraft mass and wing span of generator and follower aircraft as these are the driving factors that influence the benefit gained by the follower aircraft. Recent studies showed a potential fuel saving maximum of about $10 \%$ with identical aircraft types as generator and follower [8-12]. Hence, fuel savings at the magnitude of $20 \%$ can be regarded as realistic considering the large mass differences between A340 (190 tons) as generator and A320 (70 tons) as follower aircraft. Indeed, the exact figures cannot be validated here but the qualitative findings still hold.

The spatial distribution of the Roll Control Ratio as a measure for the rolling moment impact is depicted in Fig. 7. For an easier comparison to Fig. 6 the sweet spot position is depicted here again (red dot).

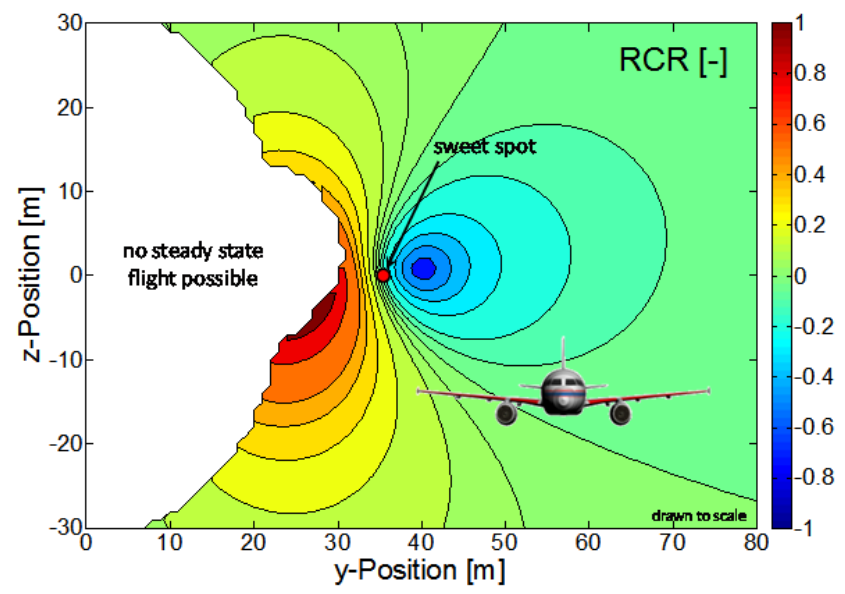

Fig. 7 Spatial distribution of Roll Control Ratio with straight vortices (A320-type $2 \mathrm{~nm}$ behind A340-type at FL370, Ma 0.78)

Fig. 7 shows that the sweet spot is located in a region with nearly no induced rolling moment, but with large gradients of the induced rolling moment to either side. This was also observed for aircraft pairings of the same aircraft type [12]. The region with almost no induced rolling moment is relatively narrow and about $5 \mathrm{~m}$ to the left and the right of this region significant rolling moments are induced. At the safe side (towards a lateral position of about $40 \mathrm{~m}$ ) the $R C R$ increases to about 0.5 , which corresponds to a necessary aileron / roll spoiler deflection of $50 \%$. In the direction towards the vortices (towards smaller ypositions) the rolling moment increases quickly and finally exceeds the follower aircraft's roll authority (if $R C R>1$, no stable flight is possible). This area has to be avoided by all means.

The simulation results shown in Fig. 6 and Fig. 7 are comparable to all previous investigations (such as those of Kaden [12] depicted in Fig. 2). Hence, up to this point, the 
method of quasi-2D analysis is considered state-of-the-art. It is well known, that the sweet spot region with the maximum fuel saving is quite narrow, but fortunately the rolling moment is relatively small in the sweet spot region. However, the rolling moment increases significantly to both sides of the sweet spot. For this reason, the accuracy of any formation flight controller in holding the relative position to the vortices is required to be very high.

\subsection{Realistically perturbed vortices}

The analysis described in section 3.1 applies a quasi2D flow field, which is invariant in longitudinal direction. As described in section 2.2, however, wake vortices cannot be assumed to be perfectly straight under real flight conditions. Slight perturbations of the vortex core positions are very likely due to atmospheric disturbances, such as turbulence, light wind shear or instabilities of the wake (for greater vortex ages).

For this reason further simulations were performed with the LES-generated flow fields described in section 2.2. Fig. 8 shows the fuel saving in the same way like Fig. 6 but for four different longitudinal positions within the LES flow field (with $\Delta x=100 \mathrm{~m}$ ). One can observe in Fig. 8 that the sweet spot position varies within a range of about $5 \mathrm{~m}$ in vertical and lateral direction. The same applies for the distribution of the Roll Control Ratio shown in Fig. 9, which varies in the same order of magnitude.

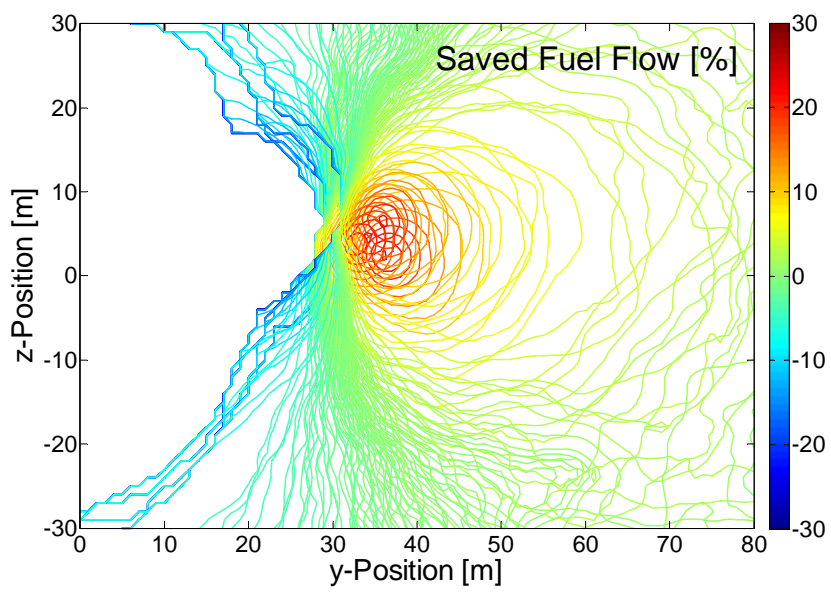

Fig. 8 Spatial distribution of fuel flow reduction with perturbed vortices from LES at four longitudinal positions with $\Delta x=100 \mathrm{~m}$ (A320-type $16 \mathrm{~s}$ behind A340-type at FL370, Ma 0.78)

Flying in this windfield results in a permanent variation of the induced rolling moment and lift. With an airspeed of about $230 \mathrm{~m} / \mathrm{s}$ (Ma 0.78 at FL370) the four different flow field slices shown in Fig. 7 with $\Delta x=100 \mathrm{~m}$ are met with a rate of about $0.4 \mathrm{~s}$. The medium and long term consequences of this rapid variation in induced forces and moments need to be compensated by the formation flight controller. For reasons of passenger comfort, the agility of such a controller must not be too high. On the other hand, the controller must prevent to encounter those areas where the impact of the wake cannot be counteracted. With a windfield that is invariant in longitudinal direction an accurate position keeping is much easier than with a changing windfield.

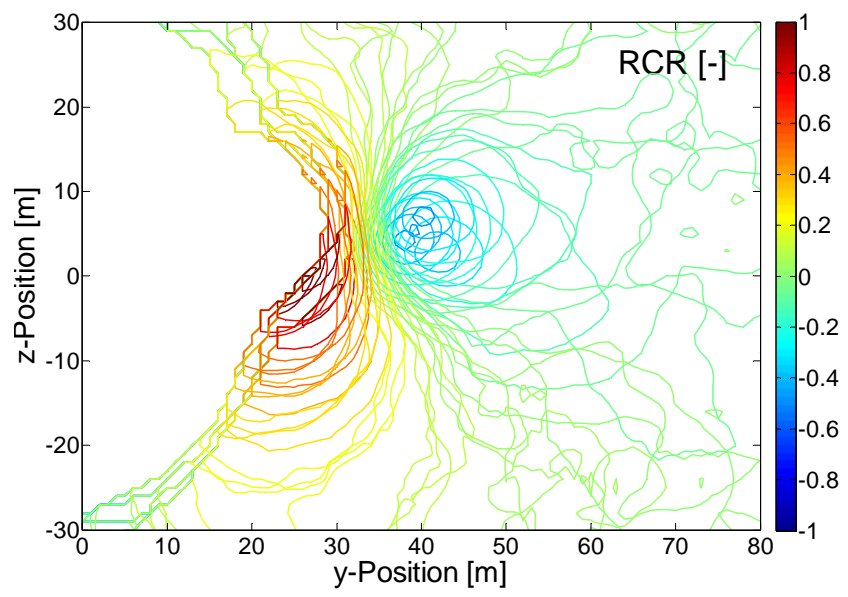

Fig. 9 Distribution of Roll Control Ratio with perturbed vortices from LES (A320-type $16 \mathrm{~s}$ behind A340-type at FL370, Ma 0.78)

In order to evaluate the possible control effort for a formation keeping controller that is still to be designed, preliminary simulations were performed using a basic flight controller, such as the so-called normal law of the A320, and a regular autopilot. As described in section 2.1 the flight control laws and the autopilot of the ATRA simulation were developed by DLR, following the architecture and dynamics of the A320 flight control system and autopilot. For the simulations the follower aircraft was trimmed in the wake's flow field at different positions relative to the vortices with the same track of the leader aircraft and without wind.

Fig. 10 shows the flight paths during 6 seconds with the normal law of the basic flight controller active and without autopilot. The normal law only compensates roll disturbances but does not hold the altitude or azimuth of the aircraft. In longitudinal motion the normal law controls the vertical load factor and without any control intput by a pilot the vertical load factor is held at a value of $1 \mathrm{~g}$. Therefore, it is not surprising that with the fluctuating 
disturbance from the perturbed vortices the aircraft does not keep its position relative to the vortices.

One can see in Fig. 10 that the flight path starting at the nearest point to the vortex (at a lateral distance of $y=30 \mathrm{~m}$ ) encounters the area where steady flight is not possible. The aircraft rapidly gains bank angle up to $20^{\circ}$ and loses about $25 \mathrm{~m}$ altitude within the 6 seconds. All other flight paths from the starting points, which are further away from the vortices, do not encounter the area where steady flight is impossible. These flight paths lead even further away from the vortices. However, these simulations only give an indication of the risk to unintentionally encounter the area where no steady flight is possible. Also, these simulations show that formation flight using manual control of the pilot is probably hard to achieve, given the quick deviations from the starting position.

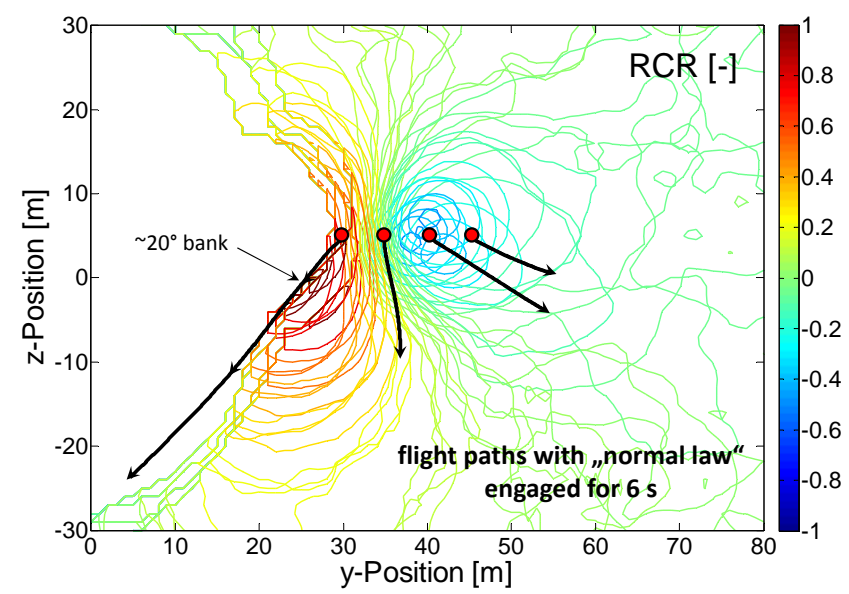

Fig. 10 Flight paths with normal law active during 6 seconds flight in vicinity to perturbed vortices (LES) with different start positions (red dots) - A320-type $16 \mathrm{~s}$ behind A340-type at FL370, Ma 0.78

In order to further analyse the controlled behaviour of the aircraft in the vicinity of the wake vortices dynamic simulation with autopilot engaged in altitude-hold and track-hold mode, again without wind, were performed. Fig. 11 shows the resulting flight paths in the three-dimensional flow field over a period of time of $300 \mathrm{~s}$ (significantly longer than in Fig. 10).

One can observe in Fig. 11 that from all of the three different starting positions the aircraft remains inside a relatively small domain even without a dedicated formation flight controller, which holds the aircraft at an exact relative position to the vortices. Even starting at the most inner position, which is quite close to the area of uncontrollable vortex impact, the aircraft does not encounter the hazardous area. The two other starting points result in a flight path that leads the aircraft away from the vortices, hence into a safe direction. The most inner starting point is indeed an operational less relevant position, as it is too close to the area where steady-state flight is impossible to be a desirable flight position for formation flight. However, an aircraft could unintentionally encounter this region close to the vortices due to external disturbances or the vortex line could be perturbed due to wind shear etc. For this reason the simulation results starting from the most inner point are explained in more detail as it could be considered as the most hazardous case.

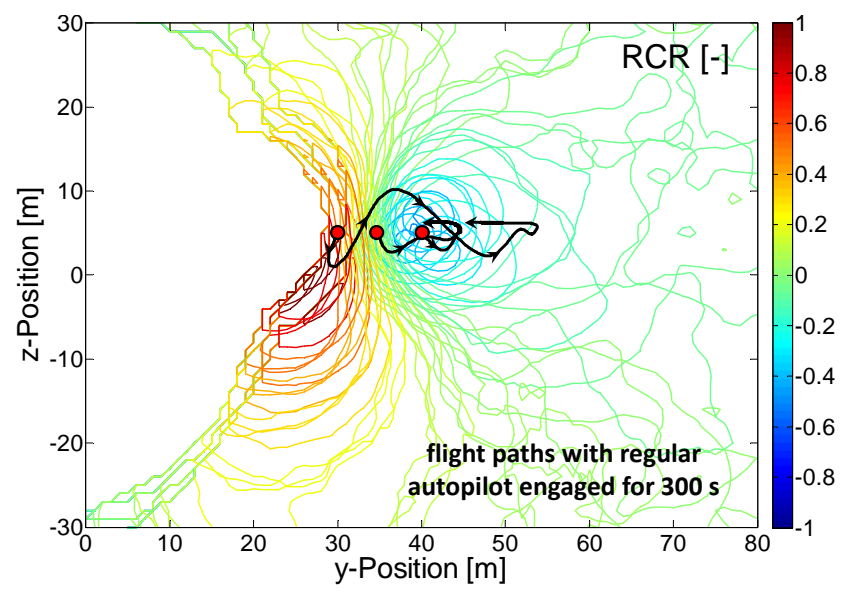

Fig. 11 Flight paths with regular autopilot during 300 seconds flight in vicinity to perturbed vortices (LES) with different start positions (red dots) - A320-type $16 \mathrm{~s}$ behind A340-type at FL370, Ma 0.78

Fig. 11 shows that without a lateral position control and with only a track-hold-controller active, the aircraft drifts sideways within the flow field. This drift occurs due to the fact that the aircraft is not perfectly at the same altitude as the vortex core, so that side-forces act on the aircraft. Without a dedicated control of the relative position within the flow field the track-hold controller is not able to compensate this drift. However, the drifting occurs relatively slowly. To illustrate this, Fig. 12 shows the lateral and vertical position of the flight path starting from the most inner position in Fig. 11 on a time scale.

Fig. 12 shows that the drift from the start position to a lateral position of about $55 \mathrm{~m}$, which can be observed in Fig. 11, takes place during the first 30 to 40 seconds, whereas the rest of the time the aircraft drifts back towards the sweet spot very slowly. In order to give in indication of the control effort of the autopilot Fig. 13 shows the aileron deflection $\xi$ and the resulting bank angle $\Phi$ corresponding to the flight path which started from the most inner position in Fig. 11. 

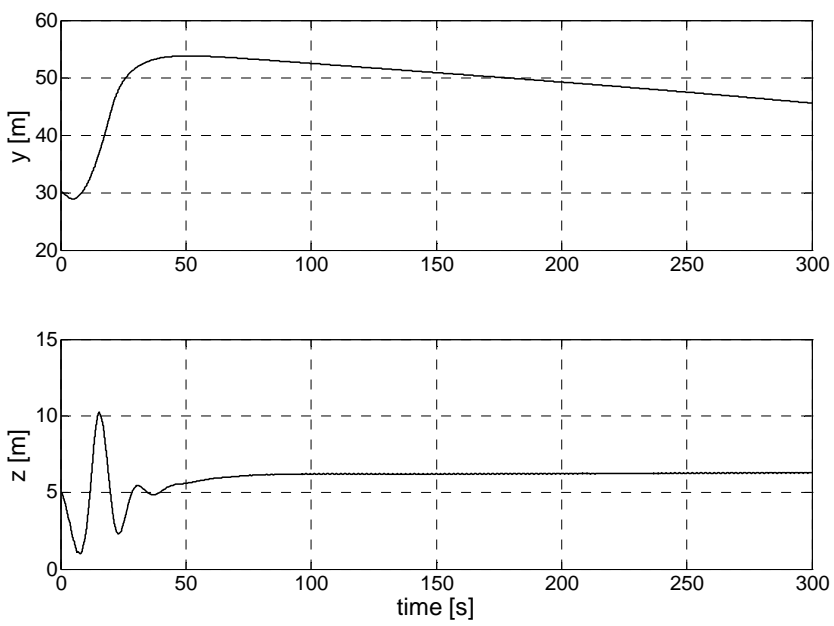

Fig. 12 Lateral and vertical position with regular autopilot during 300 seconds flight in vicinity to perturbed vortices (LES) from most inner start position (s. Fig. 11) - A320type 16 s behind A340-type at FL370, Ma 0.78

Fig. 13 shows a trimmed aileron deflection at the start of the simulation of almost $25^{\circ}$ (trimmed deflection: $\xi_{0}=24.56^{\circ}$ ), which corresponds to almost full deflection (maximum aileron deflection of A320 is $25^{\circ}$ [15]). This can also be observed in Fig. 11, where the most inner starting point lies in an area with $R C R$ of almost 1 . The figure also clearly shows the change of sign of the induced rolling moment, hence aileron deflection, at about 15 s after simulatioun start when the aircraft passes from the red to the blue area in the lower plot of Fig. 11.
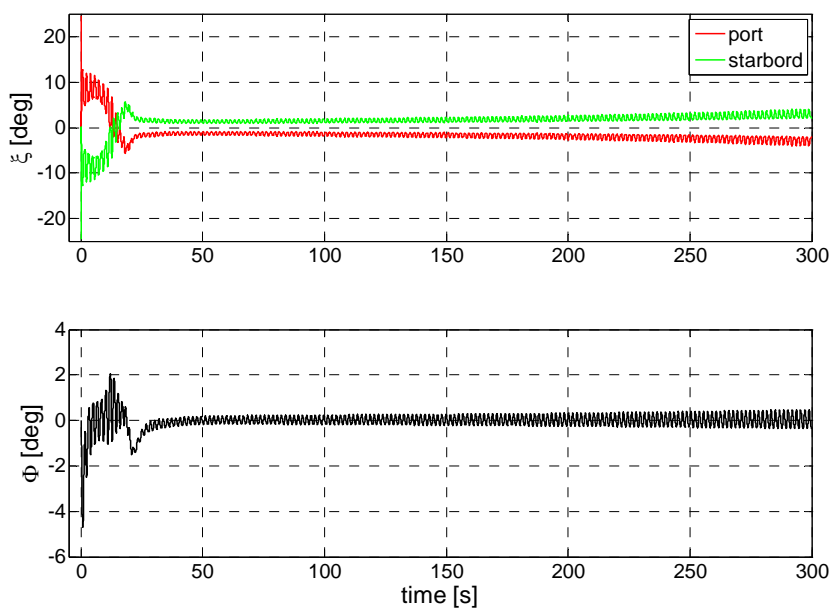

Fig. 13 Aileron deflection $\xi$ and bank angle $\Phi$ during 300 seconds flight in vicinity to perturbed vortices (LES) from the most inner start position (s. Fig. 11) - A320-type $16 \mathrm{~s}$ behind A340-type at FL370, Ma 0.78
The fast oscillation which can be observed in Fig. 13 is produced by the periodical repetition of the flow field. As described in section 2.2 the LES flow field has a length of $400 \mathrm{~m}$. With a true airspeed of about $230 \mathrm{~m} / \mathrm{s}$ (Ma 0.78 at FL370) this results in a repetition rate of about $1.7 \mathrm{~s}$. This is exactly the rate of the oscillation seen in Fig. 12. Hence, the fast oscillation is obviously produced by the fluctuation of the flow field, whereas the lower frequent movement of the ailerons is a result of the changing position of the aircraft within the wake's flow field.

It must be emphasised that the simulation used here only represents the rigid-body motion of the encountering aircraft. Structural and aeroelastic response of the aircraft due to the fluctuating vortex induced forces is neglected. Therefore, it does not make sense to analyse accelerations and load factors for investigation of passenger comfort etc. as in reality structural damping would reduce peak loads. For this reason, the authors refrain from showing load factors or accelerations here.

From the flight dynamics point of view the results of the simulations described above show that the control effort even in a three-dimensional flow field with slightly perturbed vortices is manageable as the required deflections are far below the maximum deflections. This implies that it could be sufficient to design a formation keeping controller as outer loop of the existing autopilot without the need for a dedicated formation flight controller. Such an outer loop would generate command values for altitude and track based on the actual position of the vortices, which are then controlled by the regular autopilot.

\section{Future work}

The work presented in this paper is the first step towards a realistic application of formation flight for transport aircraft. For the future further investigation will be performed at DLR Institute of Flight Systems in the area of formation flight.

This will include the development of a specific formation control system. Besides an improved position tracking performance, based on different sensor data, during the formation keeping, this controller should also allow an adequate capture of the formation. In order to enable enhanced position tracking suitable concepts for the identification of the desired flight position during the formation flight will be investigated.

Furthermore, the formation flight simulation scenarios will be investigated in the motion-based AVES simulator [33]. In a first step, a new online sweet spot detection algorithm is to be tested. These tests include manually flown simulator trials using adapted cockpit displays for im- 
provement of the situational awareness of the pilots during formation flight. As a second step, the sweet spot detection and formation flight controller design shall be combined for automatic positioning in the wake in order to achieve the best compromise between fuel saving and safety.

\section{Conclusions}

As a first step of investigation on formation flight at DLR Institute of Flight Systems, simulations of formation flight were performed, applying (ideally) straight vortices as well as more realistic flow fields generated with largeeddy-simulations (LES). These LES-generated vortex flow fields show small perturbations of the vortex lines, hence result in a flow field, which is variable in longitudinal direction. For this reason, the flow field used here can be considered as more representative for real flight conditions than those used in past studies.

First dynamic simulations with a regular autopilot engaged in altitude-hold and track-hold mode showed that even without a dedicated formation-keeping-controller it was possible to keep the aircraft in the beneficial areas of the wake. The hazardous areas in which the wake's impact is too strong to be counteracted were not encountered accidentally. For present aircraft the usage of the track hold mode is relatively untypical as usually the heading hold mode is used in airline practice. Nevertheless, for the autopilot it makes no difference if track or heading is held. For this reason the results presented here imply that it could be sufficient to design a dedicated formation flight controller as outer loop of the regular autopilot.

The models used in the study originate from previous wake encounter severity analyses. The models for the vortex flow fields as well as the aerodynamic interaction model, which calculates the vortex impact acting on the follower aircraft, were validated by means of real flight test data conducted in the framework of wake encounter research. For this reason the results presented here can be considered reliable in terms of fuel savings and vortex impact, although they cannot be validated directly. For this, flight tests with the respective aircraft pairing would be required. However, the paper's intention is not to give quantitative numbers on the exact fuel savings but to evaluate flight dynamics and flight control capabilities for formation flight. The results presented here appear promising regarding the complexity of necessary aircraft modifications in order to allow save formation flight with commercial transport aircraft.

In the future a dedicated formation flight controller law designed as outer loop to the regular A320 autopilot will be developed. With this system automated formation flight will be further investigated in the A320 motion-based simulator at the AVES simulator centre of DLR Institute of Flight Systems.

\section{References}

[1] N. N., Procedures for Air Navigation Services, Air Traffic Management, ICAO Doc 4444, 15th edition, 2007.

[2] D. Hummel, Aerodynamic Aspects of Formation Flight in Birds, J. theor. Biol. 104 (1983) 321-347.

[3] C. J. Cutts, J. R. Speakman, Energy savings in formation flight of pink-footed geese, J. exp. Biol. 189 (1994) 251-261.

[4] P. B. S. Lissamann, C. A. Shollenberger, Formation Flight of Birds, Science, Vol. 168, No. 3934 (1970), p. 1003.

[5] D. Hummel, Die Leistungsersparnis beim Verbandsflug (english: the power saving during formation flight), J. Orn. 114 (1973) 259-282.

[6] M. Beukenberg, D. Hummel, Aerodynamics, Performance and Control of Airplanes in For-mation Flight, 17th Congress of the International Council of Aeronautical Sciences (ICAS), Stockholm, Sweden, 1990.

[7] W. Blake, D. Multhopp, Design, Performance and Modeling Considerations for Close Formation Flight, AIAA Atmospheric Flight Mechanics Conference and Exhibit, AIAA 1998-4343, August 1998, pp. 476-486.

[8] M. G. Wagner, et al., Flight test results of close formation flight for fuel savings, AIAA Atmospheric Flight Mechanics Conference and Exhibit, AIAA 2002-4490, August 2002.

[9] M. J. Vachon, et al., F/A-18 aircraft performance benefits measured during the autono-mous formation flight project, AIAA Atmospheric Flight Mechanics Conference and Exhibit, AIAA 2002-4491, August 2002.

[10] S. R. Bieniawski, et al., Summary of Flight Testing and Results for the Formation Flight for Aerodynamic Benefit Program, AIAA 2014-1457, AIAA SciTech, 52nd Aerospace Sciences Meeting, national Harbor, Maryland, USA, 13-17 January 2014.

[11] J. P. Slotnick, et al., Computational Aerodynamic Analysis for the Formation Flight for Aerodynamic Benefit Program, AIAA 2014-1458, AIAA SciTech, 52nd Aerospace Sciences Meeting, national Harbor, Maryland, USA, 13-17 January 2014. 
[12] A. Kaden, R. Luckner, Modeling Wake Vortex RollUp and Vortex-Induced Forces and Moments for Tight Formation Flight, AIAA Modeling and Simulation Technologies Conference, AIAA 2013-5076, August 2013.

[13] C. Raab, Flugdynamisches Simulationsmodell A320ATRA - Validierungsversuche und Bewertung der Modellgüte (english: Flight Dynamics Simulation Model A320-ATRA - Validation Tests and Rating of the Model Accuracy), DLR Internal Report IB 1112012/43, Braunschweig, Germany, 2012.

[14] N.N., Manual of Criteria for the Qualification of Flight Simulation Training Devices, ICAO Doc-9625, (3), 2009.

[15] N.N., A320 Flight Crew Operating Manual, Part I, System Description, Issue 01-Dec-2008.

[16] N.N. A320/A321 Aircraft Maintenance Manual AMM, reference DG. AMM AEF, Issue 01-May2009.

[17] T. Gerz, C. W. Schwarz, The DLR Project Wetter und Fliegen, DLR Research Report 2012-02, ISSN 1434-8454, Oberpfaffenhofen, Germany, 2012.

[18] D. Fischenberg, Bestimmung der Wirbelschleppencharakteristik aus Flugmessdaten (english: Determination of Wake Vortex Characteristics from Flight Test Data), German Aerospace Congress, Stuttgart, Germany, 2002.

[19] D. Burnham, J. Hallock, Chicago Monostatic Acoustic Vortex Sensing System 4, Wake Vortex Decay, National Information Service, Springfield, Virginia, USA, 1982.

[20] L. Rosenhead, The Formation of Vortices from a Surface of Discontinuity, Proceedings Royal Society of London, Ser. A., 134: p.170-192, 1932.

[21] F. Holzäpfel, Probabilistic Two-Phase Wake Vortex Decay and Transport Model, Journal of Aircraft, Vol. 40, No. 2 (2003) 323-331.

[22] D. Vechtel, Simulation study of wake encounters with straight and deformed vortices, Aeronaut J, April 2016, 120, (1226).

[23] T. M. Barrows, Simplified Methods of Predicting Aircraft Rolling Moments due to Vortex Encounters, AIAA Paper 76-61, AIAA 14th Aerospace Sciences Meeting, Washington DC, USA, 1976.

[24] A. de Bruin, WAVENC - Wake Vortex Evolution and Wake Vortex Encounter, Publishable Synthesis Report, National Aerospace Lab., NLR-TR-2000-079, Amsterdam, The Netherlands, 2000.

[25] R. Jategaonkar, D. Fischenberg, W. v. Gruenhagen, Aerodynamic Modelling and System Identification from Flight Data - Recent Applications at DLR, J Aircr, 2004, 41, (4), p 687.
[26] D. Vechtel, Inflight simulation of wake encounters using deformed vortices, Aeronaut J, October 2013, 117, (1196).

[27] D. Vechtel, Flight simulator study on the influence of vortex curvature on wake encounter hazard using LES wind fields, Aeronaut J, March 2012, 116, (1177).

[28] D. Fischenberg, A method to validate wake vortex encounter models from flight test data, ICAS 2010, 27th International congress of the aeronautical sciences, Nice, France, 2010.

[29] C. W. Schwarz, K.-U. Hahn, Full-flight simulator study for wake vortex hazard area investigation, Aerospace Science and Technology, 2006, 10, (2), pp 136-143.

[30] K.-U. Hahn, C. W. Schwarz, Safe Limits for Wake Vortex Penetration, AIAA Paper 2007-6871, AIAA Guidance, Navigation and Control Conference and Exhibit, Hilton Head, South Carolina, USA, 2007.

[31] C. W. Schwarz, D. Vechtel, Wake Vortex Encounter Severity Criteria for RECAT, DLR Internal Report IB 111-2012/44, Braunschweig, Germany, 2012.

[32] S. C. Crow, Panel Discussion, Symposium on Aircraft Wake Turbulence, Seattle, Washington, US, 1-3 September 1970.

[33] H. Duda, et al., Design of the DLR AVES Research Flight Simulator, AIAA Modeling and Simulation Technologies Conference, AIAA 2013-4737, August 2013. 